\title{
Analyzing UHECR arrival directions through the Galactic magnetic field in view of the local universe as seen in $2 \mathrm{MRS}$
}

\section{Markus Ahlers}

Niels Bohr International Academy

\section{Peter Denton}

Niels Bohr International Academy

\section{Mohamed Rameez*}

Niels Bohr International Academy

E-mail: Mohamed.Rameez@nbi.ku.dk

\begin{abstract}
We examine the distribution in the arrival directions of the highest energy cosmic rays observed by the Pierre Auger Observatory and Telescope Array using spherical harmonics. We take advantage of the highly anisotropic distribution of matter within the Greisen-Zatsepin-Kuzmin horizon as observed by the 2MASS Redshift Survey coupled with the relatively small deflections expected for protons from current models of the Galactic magnetic field to constrain the likelihood of different nuclear compositions of the UHECR flux.
\end{abstract}

35th International Cosmic Ray Conference - ICRC2017

10-20 July, 2017

Bexco, Busan, Korea

\footnotetext{
${ }^{*}$ Speaker.
} 


\section{Introduction}

The sources of Cosmic Rays (CRs) have not been identified. Being charged particles, their arrival directions do not point back to their sources since they are deflected by magnetic fields on their way to Earth. At energies of tens of EeV, the deflections due to the Galactic magnetic fields are expected to be a few degrees [1] for protons and light nuclei. The relative isotropy of the arrival directions of the events at these energies as reported by the Pierre Auger Observatory [2] and Telescope Array (TA) [3] and lack of correlation with Galactic structure [4] hint at a predominantly extragalactic origin for these events.

CRs above $\sim 50 \mathrm{EeV}$ cannot propagate more than a few hundred Mpcs due to the resonant enhancement in their interaction cross section with Cosmic Microwave Background (CMB) photons [5, 6]. Astronomical surveys of the Universe within this horizon, such as the 2MASS Redshift Survey (2MRS) [7] (see section 2) have found the matter distribution to be highly anisotropic.

In this work we study how such an anisotropic matter distribution can be connected to the relatively isotropic distribution of events observed at Earth, as reported by Auger and TA. We consider a population of sources of equal luminosity that follow the matter distribution of the local Universe as observed in 2MRS and simulate sky-maps of the expected arrival directions. Propagation effects in both extragalactic space (see section 4) and and through the Galactic magnetic field (see section 5) are accounted for. The anisotropy expected in $N$ events following these maps is quantified in section 6. The process is repeated for different scenarios of source density $\left(10^{-3}-10^{-5} \mathrm{Mpc}^{-3}\right)$, spectral index at source $(\Gamma=0.9,2$, see [8]), composition at source ( $p, S i$, or $F e$ ) and extragalactic magnetic field strengths and subsequently compared with the level of anisotropy observed in data to quantify the compatibility between these scenarios and observations. Finally, in section 7 we present our results and conclusions.

\section{The local universe as seen in the 2MASS Redshift Survey}

The 2MASS Redshift Survey (2MRS) [7] maps the distribution of galaxies and dark matter out to a mean redshift of $z=0.03$ ( $\sim 120 \mathrm{Mpc}$ for a pure Hubble flow). It is the densest all-sky redshift survey to date and contains $\sim 45,000$ galaxies to a limiting $\mathrm{K}$ band magnitude of 11.75 . The distribution of the directions of these galaxies within different redshift slices is highly anisotropic (see figure 1). Clear excesses can be seen in the direction of structures such as the Large Magellanic Cloud, the Supergalactic plane and the Virgo cluster.

\section{Simulating maps}

A mock catalog of UHECR sources that follow the 2MRS matter distribution can be generated by the following process. For a given source density, the number of sources expected within a sphere of radius $600 \mathrm{Mpc}$ around the Galaxy is calculated. For these sources, redshifts are generated according to a $(1+z)^{3}$ source evolution scenario (very similar to the star formation rate [9]). For each of these redshifts, a right ascension (RA) and declination $(\delta)$ are generated by sampling from a healpix [10] map of nside 32, constructed with 2MRS sources in a shell of width $2 \mathrm{Mpc}$ around the comoving radius of the corresponding redshift. 

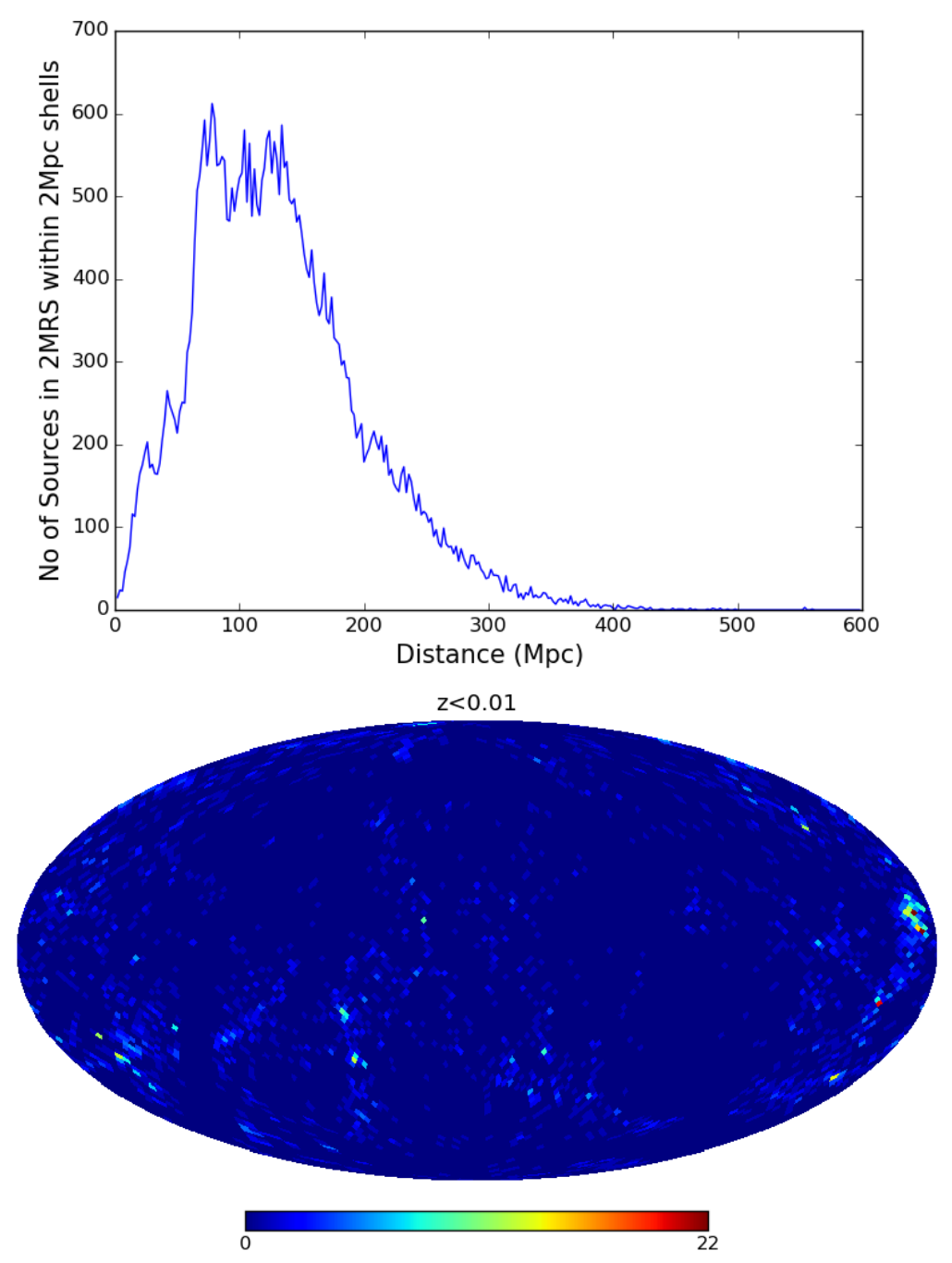

Figure 1: Top: The number of sources per spherical slice of radial width $2 \mathrm{Mpc}$, in the $2 \mathrm{MRS}$ catalog as a function of distance, assuming purely Hubble flow. Initially, this scales with the volume. Beyond $\sim 120 \mathrm{Mpc}$ $(z=0.03)$ beyond which $2 \mathrm{MRS}$ is not complete, it falls off. Bottom: The angular distribution of sources at redshift less than 0.01 .

\section{Extragalactic propagation and deflections}

We use 1D simulations in CRPropa3 [11] to estimate the evolution of composition and energy of the particles while propagating in extragalactic space. We account for photopion production, electron pair production and photo disintegration effects (for heavier nuclei) off both CMB and the Infrared Background (IRB). We also keep track of the evolution of spectrum and composition at different distances and the decay of unstable nuclei.

Deflections due to extragalactic magnetic fields are estimated as described in [12]. The deflection angle for a nucleus of atomic number $Z$ and energy $E$, propagating a distance $D$ in a magnetic 
field $B_{\text {exgal }}$ with correlation length $\lambda$ is given by,

$$
\theta_{\text {def }}=0.025^{\circ}(D / \lambda)^{1 / 2}(\lambda / 10 \mathrm{Mpc})\left(B_{\text {exgal }} / 10^{-11} \mathrm{G}\right)\left(E / 10^{20} \mathrm{eV}\right)^{-2} Z,
$$

where we take $\lambda=10 \mathrm{Mpc}$ as the characteristic correlation length.

Deflections due to extragalactic magnetic fields are expected to be random, and are consequently modeled by Gaussian beam smoothing of the maps of different radial distance slices, proportional to the expected deflection from the slice for a given composition and injection spectrum hypothesis. CRs from further away sources are expected to be deflected more, and consequently further away maps are smoothed by larger deflection angles.

Using the above method, for a mock catalog of sources as described in section 3, the expected arrival direction map before Galactic deflections can be constructed. Figure 2, left illustrates such a map.

\section{Deflections in the Galactic magnetic field}

Ref. [1] provides a 3-dimensional map of the magnetic field of the Galaxy, compiled from Faraday-rotation measurements. We employ a strategy of back propagation to estimate the impact of deflections in the Galactic magnetic field. The Galaxy is modeled as a sphere of radius $20 \mathrm{kpc}$ and a large number of CRs are isotropically injected from the Earth $8.5 \mathrm{kpc}$ from the Galactic Center. The simulated particles are given a negative charge, in order to emulate an equivalent forward propagating particle of positive charge. The direction of the particle as it exits the Galactic sphere is kept track of.

The map of expected arrival directions at Earth can subsequently be created by constructing a map from the injection directions of the above sample of events, in such a way that each event is weighted by the value corresponding to its direction as it exits the Galactic sphere obtained from the map as described in section 4. Events are also weighted according to their energy and charge, to account for arrival direction dependent differences in spectrum and composition for each mock catalog map. Figure 2, right illustrates a map constructed by the above process, corresponding to the same map without Galactic magnetic field deflections illustrated in figure 2, left.
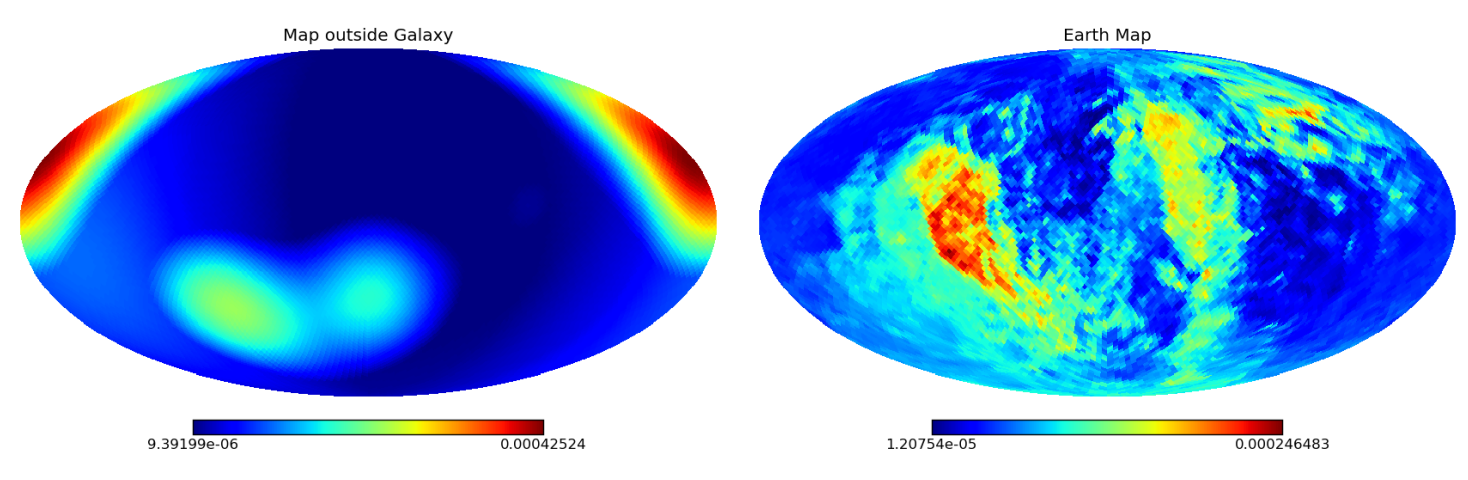

Figure 2: Left: Arrival distribution distribution without Galactic magnetic field deflections. Right: the left map with Galactic magnetic field deflections. The initial conditions here are pure iron injected at the source, spectral index of $\Gamma=-0.9, B_{\text {exgal }}=10^{-9} \mathrm{G}$, and $10^{-4} \mathrm{Mpc}^{-3}$ source density. 


\section{Likelihood quantifier of anisotropy}

We consider two different approaches to determining the compatibility of the data with the various simulations. The first involves comparing the data directly with the simulations and benefits from including all the details of the data. The next involves comparing the observed (very low) anisotropy with the simulated anisotropy via spherical harmonics. This approach benefits from being less sensitive to small perturbations in Galactic magnetic field and the other parameters.

Terrestrial experiments cannot exactly reconstruct anisotropies [13] due to the non uniform exposure $\omega(\delta)$ of the experiments [14]. By combining data from Auger and TA, with the appropriate fudge factor described in [15], the true map at the Earth can be determined.

\subsection{Likelihood Function}

The most straightforward way of comparing a given distribution to data is to calculate the likelihood. For a map $\Phi$, the likelihood is,

$$
\mathscr{L}=\prod_{i=1}^{N} \frac{\Phi\left(\mathbf{u}_{i}\right)}{\omega\left(\mathbf{u}_{i}\right)}
$$

We then find the maximum likelihood $\mathscr{L}_{0}$ over the parameters scanned and use the test statistic $-2 \log \frac{\mathscr{L}}{\mathscr{L}_{0}}$ that behaves like $\Delta \chi^{2}$.

\subsection{Spherical Harmonics}

An alternative statistical approach that is insensitive to small scale differences is spherical harmonics. Spherical harmonics, $Y_{\ell m}(\theta, \phi)$, are an orthogonal set of functions on the sphere. Any function $I(\theta, \phi)$ can be expanded in $Y_{\ell m}$ 's with coefficients $a_{\ell m}$. The rotationally invariant power spectrum, $C_{\ell}$, at a given $\ell$ is then the average of the $\left|a_{\ell m}\right|^{2}$ s.

For $N$ events distributed isotropically, the angular power spectrum has mean and standard deviation,

$$
\bar{C}_{\ell}=\frac{1}{4 \pi N}, \quad \sigma_{\ell}=\frac{1}{4 \pi N} \sqrt{\frac{N-1}{N} \frac{2}{2 \ell+1}},
$$

as derived in appendix A. We then construct the $\chi^{2}$ test statistic in the usual way by summing up through $\ell_{\max }$.

To determine $\ell_{\max }$ in a systematic way, we use the method described in [16] by counting the number of nodal zones - regions of $Y_{\ell m}$ that all have the same sign. The average (over $m$ ) number of nodal zones at a given $\ell$ is,

$$
\left\langle N_{Z}(\ell, m)\right\rangle_{m}=\frac{\ell+1}{3(2 \ell+1)}\left(2 \ell^{2}+4 \ell+3\right) .
$$

For the 204 UHECRs in the data set, this maps onto $\ell_{\max }=23$.

We then generate events according to a given map convolved with the detector's exposure, and then reconstruct the power spectrum by weighting each event with $\omega^{-1}(\delta)$. 
Table 1: Left: The fraction of simulations with a small $\chi^{2}$ than the data. Note that for protons there was no random sampling of the maps that lead to a $\chi^{2}$ more isotropic than the data. Right: The same but varying $B_{\text {exgal }}$ for iron composition.

\begin{tabular}{c|c}
$Z$ & $p$ \\
\hline 1 & $<4.2 \times 10^{-5}$ \\
14 & $1.8 \times 10^{-3}$ \\
26 & $4.5 \times 10^{-2}$
\end{tabular}

\begin{tabular}{c|c}
\multicolumn{2}{c}{$\mathrm{Fe}$} \\
\hline$B_{\text {exgal }} \mathrm{G}$ & $p$ \\
\hline $10^{-11}$ & $1.6 \times 10^{-2}$ \\
$10^{-9}$ & $7.3 \times 10^{-2}$
\end{tabular}

\section{Results and Conclusions}

Using the likelihood method we find that iron, $\Gamma=2, \rho=10^{-3} \mathrm{Mpc}^{-3}, B_{\text {exgal }}=10^{-9} \mathrm{G}$ is the best fit point. A proton composition is disfavored at $\sigma=\sqrt{-2 \log \frac{\mathscr{L}}{\mathscr{L}_{0}}}=13$.

For the spherical harmonic test, we first calculate the power spectrum of the data up to $\ell_{\max }=$ 23 and see that it is consistent with isotropic as shown in figure 3 with $\chi^{2} /$ dof $=30.6 / 22$ which corresponds to a $p$-value of 0.104 confirming that the data is consistent with isotropic and that comparing simulated maps with a test statistic built on the null hypothesis of isotropy is a reasonable assumption. We show the distribution of $\chi^{2}$ 's for some representative cases in figure 3 and summarize our results in table 1 . We note that for a pure proton composition the data is extremely anisotropic and is strongly disfavored. Moreover, we find weak evidence for a stronger, $\sim 10^{-9} \mathrm{G}$ extragalactic magnetic field.
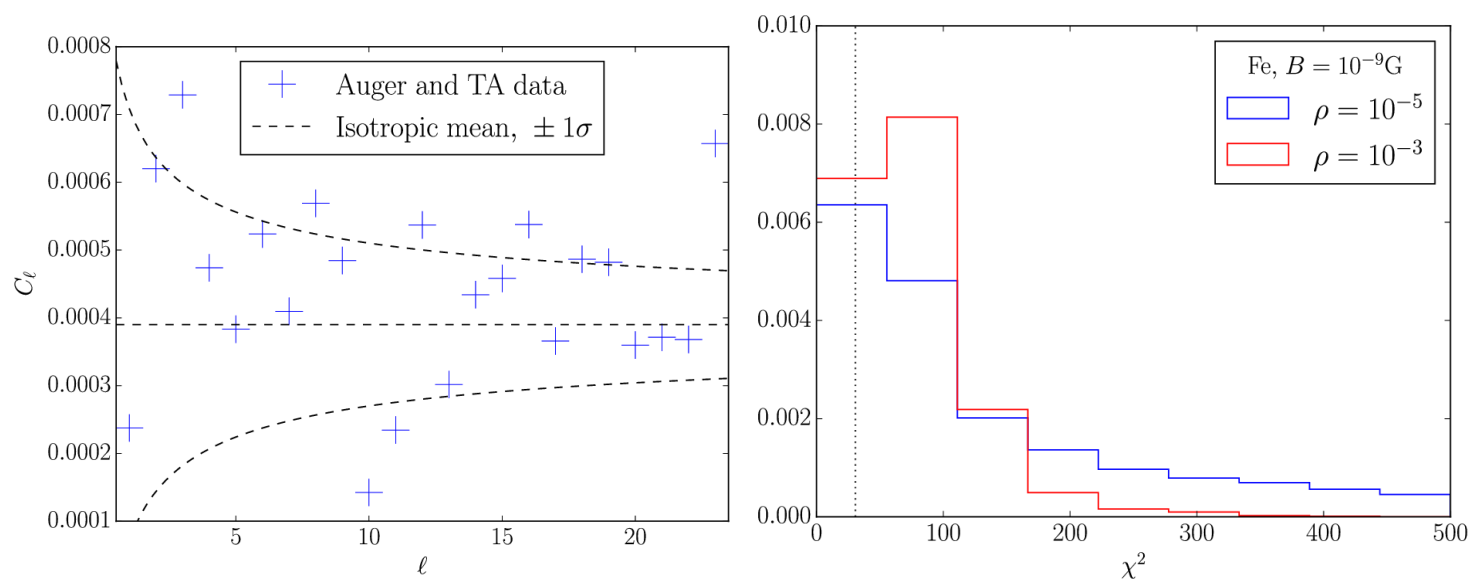

Figure 3: Left: The power in the Auger and TA data at various $\ell$ 's. The dashed lines show the mean and $\pm 1 \sigma$ spread expected from an isotropic distribution. Right: The distribution of $\chi^{2}$,s for a pure iron composition, $B_{\text {exgal }}=10^{-9} \mathrm{G}, \Gamma=2$, and with different source densities. The vertical dashed line is the $\chi^{2}$ of the data.

We conclude that pure iron at the source is consistent with data while a pure proton composition is strongly disfavored at $p<4.2 \times 10^{-5}(>4 \sigma)$ (which is only limited by computational power).

\section{Acknowledgments}

MA and MR are supported by the Danish National Research Foundation (DNRF91 and Grant 
no. 1041811001). PBD acknowledges support from the Villum Foundation (Project No. 13164), and the Danish National Research Foundation (DNRF91).

\section{A. Power Spectrum Moments}

From [17] we have that the power spectrum can be rewritten as

$$
C_{\ell}=\frac{1}{4 \pi N}+\frac{1}{2 \pi N^{2}} \sum_{i<j} P_{\ell}\left(\mathbf{u}_{i} \cdot \mathbf{u}_{j}\right)
$$

for discrete sources located at $\left\{\mathbf{u}_{i}\right\}_{i=1}^{N}$ where $P_{\ell}$ is the Legendre polynomial. We calculate the mean and standard distribution for a uniform distribution. Over a uniform distribution we have

$$
\left\langle P_{\ell}\left(\mathbf{u}_{i} \cdot \mathbf{u}_{j}\right)\right\rangle_{u}=\frac{1}{2} \int_{-1}^{1} P_{\ell}\left(\mathbf{u}_{i} \cdot \mathbf{u}_{j}\right) d(\cos \theta)=0
$$

for $\ell>0$. Then,

$$
\left\langle C_{\ell}\right\rangle_{u}=\frac{1}{4 \pi N} .
$$

For the next moment we first see that

$$
\left\langle P_{\ell}\left(\mathbf{u}_{i} \cdot \mathbf{u}_{j}\right) P_{\ell}\left(\mathbf{v}_{i} \cdot \mathbf{v}_{j}\right)\right\rangle_{u}=\frac{1}{4} \int_{-1}^{1} \int_{-1}^{1} P_{\ell}(x) P_{\ell}(y) d x d y=0,
$$

by separation since $x, y$ vary independently of each other, and

$$
\left\langle\left[P_{\ell}\left(\mathbf{u}_{i} \cdot \mathbf{u}_{j}\right)\right]^{2}\right\rangle_{u}=\frac{1}{2} \int_{-1}^{1}\left[P_{\ell}(x)\right]^{2} d x=\frac{1}{2 \ell+1},
$$

for $\ell>0$ by the normalization of the Legendre polynomials. We then write out $C_{\ell}^{2}$ dropping terms linear in $P_{\ell}\left(\mathbf{u}_{i} \cdot \mathbf{u}_{j}\right)$ and terms with the product $P\left(\mathbf{u}_{i} \cdot \mathbf{u}_{j}\right) P\left(\mathbf{u}_{k} \cdot \mathbf{u}_{\ell}\right)$ for $\mathbf{u}_{i} \cdot \mathbf{u}_{j} \neq \mathbf{u}_{k} \cdot \mathbf{u}_{\ell}$,

$$
\left\langle C_{\ell}^{2}\right\rangle_{u}=\frac{1}{16 \pi^{2} N^{2}}+\frac{1}{4 \pi^{2} N^{4}} \sum_{i<j}\left\langle\left[P_{\ell}\left(\mathbf{u}_{i} \cdot \mathbf{u}_{j}\right)\right]^{2}\right\rangle_{u} .
$$

Then the standard deviation of the power spectrum of a uniform distribution is,

$$
\sigma=\frac{1}{4 \pi N} \sqrt{\frac{N-1}{N} \frac{2}{2 \ell+1}}
$$

which we note is less than the mean for all $\ell>0$.

\section{References}

[1] R. Jansson and G. R. Farrar, The Galactic Magnetic Field, Astrophys. J. 761 (2012) L11, [1210.7820].

[2] Pierre Auger collaboration, A. Aab et al., The Pierre Auger Cosmic Ray Observatory, Nucl. Instrum. Meth. A798 (2015) 172-213, [1502.01323].

[3] Telescope Array collaboration, H. Kawai et al., Telescope array experiment, Nucl. Phys. Proc. Suppl. 175-176 (2008) 221-226. 
[4] R. Aloisio, V. Berezinsky and A. Gazizov, Transition from galactic to extragalactic cosmic rays, Astropart. Phys. 39-40 (2012) 129-143, [1211. 0494 ].

[5] K. Greisen, End to the cosmic ray spectrum?, Phys. Rev. Lett. 16 (1966) 748-750.

[6] G. T. Zatsepin and V. A. Kuzmin, Upper limit of the spectrum of cosmic rays, JETP Lett. 4 (1966) 78-80.

[7] A. C. Crook, J. P. Huchra, N. Martimbeau, K. L. Masters, T. Jarrett and L. M. Macri, Groups of Galaxies in the Two Micron All-Sky Redshift Survey, Astrophys. J. 655 (2007) 790-813, [astro-ph/0610732].

[8] Pierre Auger collaboration, A. Aab et al., Combined fit of spectrum and composition data as measured by the Pierre Auger Observatory, JCAP 1704 (2017) 038, [1612.07155].

[9] H. Yuksel, M. D. Kistler, J. F. Beacom and A. M. Hopkins, Revealing the High-Redshift Star Formation Rate with Gamma-Ray Bursts, Astrophys. J. 683 (2008) L5-L8, [0 804.4008 ].

[10] K. M. Gorski, E. Hivon, A. J. Banday, B. D. Wandelt, F. K. Hansen, M. Reinecke et al., HEALPix - A Framework for high resolution discretization, and fast analysis of data distributed on the sphere, Astrophys. J. 622 (2005) 759-771, [astro-ph/ 0409513 ].

[11] R. Alves Batista, A. Dundovic, M. Erdmann, K.-H. Kampert, D. Kuempel, G. Müller et al., CRPropa 3 - a Public Astrophysical Simulation Framework for Propagating Extraterrestrial Ultra-High Energy Particles, JCAP 1605 (2016) 038, [1603.07142].

[12] E. Waxman and J. Miralda-Escude, Images of bursting sources of high-energy cosmic rays. 1. Effects of magnetic fields, Astrophys. J. 472 (1996) L89-L92, [astro-ph/ 960705 9].

[13] JEM-EUSO collaboration, P. B. Denton, L. A. Anchordoqui, A. A. Berlind, M. Richardson and T. J. Weiler, Sensitivity of orbiting JEM-EUSO to large-scale cosmic-ray anisotropies, J. Phys. Conf. Ser. 531 (2014) 012004, [1401.5757].

[14] P. Sommers, Cosmic ray anisotropy analysis with a full-sky observatory, Astropart. Phys. 14 (2001) 271-286, [astro-ph/0004016].

[15] Pierre Auger, Telescope Array collaboration, T. Abu-Zayyad et al., Pierre Auger Observatory and Telescope Array: Joint Contributions to the 33rd International Cosmic Ray Conference (ICRC 2013), in Proceedings, 33rd International Cosmic Ray Conference (ICRC2013): Rio de Janeiro, Brazil, July 2-9, 2013, 2013. 1310.0647.

[16] P. B. Denton and T. J. Weiler, Sensitivity of full-sky experiments to large scale cosmic ray anisotropies, JHEAp 8 (2015) 1-9, [1505.03922].

[17] P. B. Denton and T. J. Weiler, The Fortuitous Latitude of the Pierre Auger Observatory and Telescope Array for Reconstructing the Quadrupole Moment, Astrophys. J. 802 (2015) 25, [1 409.0883 ]. 